\title{
Steric effects on molecular adsorption at columnar surfaces. A model for the adsorption of pyridine on gold electrodes
}

\author{
M.M. Gómez a, J.M. Vara c , J.C. Hernández a, R.C. Salvarezza ${ }^{\text {b }}$, A.J. Arvia ${ }^{\text {b,* }}$ \\ a Departamento de Química-Física Aplicada, Facultad de Ciencias, Universidad Autónoma, Madrid, Spain \\ ${ }^{\mathrm{b}}$ Instituto de Investigaciones Fisicoquímicas Teóricas y Aplicadas (INIFTA) (University of La Plata-CONICET-CIC Pcia.BsAs), Sucursal 4, \\ Casilla de Correo 16, (1900) La Plata, Argentina \\ ${ }^{\mathrm{C}}$ Visiting Professor at the University of La Plata (Chemistry Department) from Departamento de Quimica-Física Aplicada, Facultad de Ciencias, \\ Universidad Autónoma, Madrid, Spain
}

Received 10 November 1998; received in revised form 22 June 1999; accepted 6 July 1999

\begin{abstract}
Results for pyridine (Py) adsorption on columnar-structured (cs) gold substrates from acid solutions at different potentials and $298 \mathrm{~K}$ are compared to the predictions of a recently proposed Langmuirian adsorption model at columnar surfaces. The difference in the apparent adsorption Gibbs energy derived from the adsorption isotherm of Py on cs- and smooth gold substrates was accounted for by a correction factor $(f)$ that depends on the roughness $(R)$ of the substrate according to $f \propto R^{-n}$, as expected from Monte Carlo simulation of the model. This agreement indicates that the substrate geometry plays a key role in the interpretation of adsorption isotherms. (C) 1999 Elsevier Science S.A. All rights reserved.
\end{abstract}

Keywords: Adsorption at columnar surfaces; Adsorption modeling; Pyridine adsorption on gold

\section{Introduction}

The role of adsorbate-substrate interactions at irregular surfaces in affecting the interpretation of adsorption isotherms has long been recognised [1]. One of the effects is constrained to surface domains with an average size on the order of that of the adsorbate cross section [1]. In this case, adsorption involves a steric effect appearing usually as an excluded area or volume for molecular adsorption. The excluded area can be expressed by the difference between the contour of the real substrate and the contour defined by the centers of adsorbed species on the substrate [2]. The excluded area effect introduces uncertainties in both the evaluation of the true substrate active area by physical adsorption, and the interpretation of physico-chemical magnitudes derived from adsorption isotherms [1-5].

The experimental verification of pure excluded area effects turns out to be considerably difficult because of the presence of other effects such as particle-particle

* Corresponding author. Fax: + 54-2214-254642.

E-mail address: ajarvia@inifta.unlp.edu.ar (A.J. Arvia) interactions and energy barriers at different places of the irregular substrate. Both contributions would produce effects comparable to those caused by excluded areas on the adsorption isotherms. Therefore, to discriminate among these various effects theoretical approaches based upon Monte Carlo simulation schemes have been developed [6-8].

Recently, Monte Carlo simulations of a simple adsorption model have been made to determine the influence of the diameter and depth of pores present in an ideal columnar-structured (cs) 2D surface (profile) [9]. In this model 2D-condensation at pore walls has been prevented [10]. It was found that the apparent adsorption energy decreases as the pore diameter is decreased and the pore depth is increased. The difference in the adsorption isotherm parameters for cs as compared to a smooth substrate (ss) was expressed by a correction factor $(f)$ defined as the apparent adsorption Gibbs energy ratio resulting from cs and ss. The model predicts that the value of $f$ diminishes with the roughness factor $(R)$ of the substrate according to $f \propto R^{-n}, n$ changing from $1 / 5$ to 1 as the pore diameter approaches the adsorbate cross section, that is, as the excluded area 
effect increases. Therefore, the model predicts a marked influence of $\mathrm{nm}$-sized irregularities at the substrate on the experimental data derived from adsorption isotherms.

In this paper data for pyridine (Py) adsorption on columnar-structured gold electrodes are satisfactorily compared to the predictions of this simple adsorption model. From this comparison, it is concluded that the substrate geometry has a considerable influence on the characteristics of the adsorption isotherm.

\section{The model}

A full description of the model was given in reference [9]. Briefly, a square lattice was used for the two-dimensional (2D) Monte Carlo simulations. The system consisted of a cross section of a real three-dimensional (3D) solid surface (the electrode) in contact with particles confined within a reservoir (the solution).

The solid surface was represented by a continuous 2D profile involving pores of mouth diameter $d$, column width $d^{\prime}$, and depth $L$ (Fig. 1a). Values of $d, d^{\prime}$ and $L$ were changed in the ranges $2 a \leq d \leq n a, 2 a \leq d^{\prime} \leq n a$, $a \leq L \leq n^{\prime} a$, and $1 \leq d^{\prime} / d \leq 4$, and $n \leq 8$ and $n^{\prime} \leq 60$ are integers. Values of $d, d^{\prime}$ and $L$ were scaled taking the maximum diameter of the Py molecule, $a=8 \times 10^{-8}$ $\mathrm{cm}$. This molecule was used as the adsorbate for testing the model.

Particles representing adsorbable molecules in the solution were randomly distributed in the reservoir

(a)

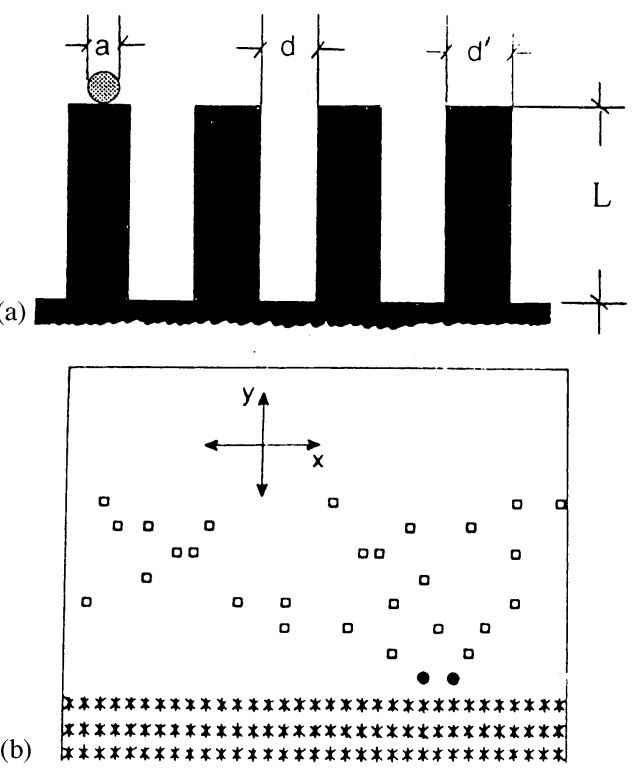

Fig. 1. (a) A 2D profile of the columnar-structured substrate. The particle used as the yardstick is included at the top of the first column. (b) Scheme of the system at the particle level. $(*)$ substrate; $(\square)$ adsorbable particles in the reservoir; ( $)$ adsorbed particles. Arrows indicate possible $(\square)$ particle displacement directions.
(Fig. 1b). No particle-particle interaction in the solution was taken into account. The movement of particles was simulated in the following way. Both a particle and one of its neighbour sites were selected at random with the same probability in the square lattice. The selected particle was allowed to jump to its neighbour site if it was unoccupied. Otherwise, the location of the particle did not change. This simulation procedure introduced a spatial random walk of particles. The Monte Carlo time step $\left(t_{\mathrm{M}}\right)$ was defined as the time required to allow all particles to move once on average.

Particles contacting the surface (Fig. 1b) were adsorbed with a probability $P_{\mathrm{s}}$ and desorbed with a probability $P_{\mathrm{d}}=1-P_{\mathrm{s}}$. Typical values of $P_{\mathrm{s}}$ were $0.999,0.98,0.95$ and 0.93 . These values of $P_{\mathrm{s}}$ account for non-negligible adsorbate-substrate interactions. In this version of the model no interaction between the adsorbed particles was considered. The surface coverage $(\theta)$ was evaluated from the snapshots as the number of adsorbed particles/number of adsorption sites ratio. Note that the definition of $\theta$ involves the 'real surface area' of the system, i.e. the entire pore area (wall and bottom) as well as the area in between pores. The simulation routine was extended until equilibrium surface coverage $\left(\theta_{\mathrm{e}}\right)$ was attained.

\section{Monte Carlo simulations}

Typical snapshots resulting from profiles with different values of $d, d^{\prime}$, and $P_{\mathrm{s}}$ at different $t_{\mathrm{M}}$ were obtained. For $d^{\prime}=4.8 \times 10^{-7} \mathrm{~cm}, d=1.6 \times 10^{-7} \mathrm{~cm}$ and $L=$ $4 \times 10^{-6} \mathrm{~cm}$ (Fig. 2a), as $t_{\mathrm{M}}$ is increased, the value of $\theta$ increases gradually leading to two distinguishable types of adsorbed particles, namely, those particles adsorbed at column tops and those adsorbed at pore walls. A detailed inspection of the snapshots reveals that, in some cases, neighbour particles adsorbed at opposite wall sites produce a plugged bottleneck effect. Blocking of the pore entrance and bottleneck effects at some pores can be seen in the snapshot shown in Fig. 2a for $\theta=0.173$. As $d$ is increased to $3.2 \times 10^{-7}$ (Fig. 2b), pore blocking tends to disappear although pore tortuosity still remains.

Data from snapshots were plotted as $\theta_{\mathrm{e}}$ versus $c$ (Fig. 3a) and adjusted according to the Langmuir equation [24],

$\theta_{\mathrm{e}} /\left(1-\theta_{\mathrm{e}}\right)=\beta c$

where $\beta$, the adsorption coefficient, which depends on the adsorption Gibbs energy $\left(\Delta G_{\text {ads }}^{\circ}\right)$. Eq. (1) predicts a linear $\theta_{\mathrm{e}} / 1-\theta_{\mathrm{e}}$ versus $c$ plot going through the origin of coordinates. This behaviour agrees with results from our Monte Carlo simulations (Fig. 3b). The slope of 
(a)
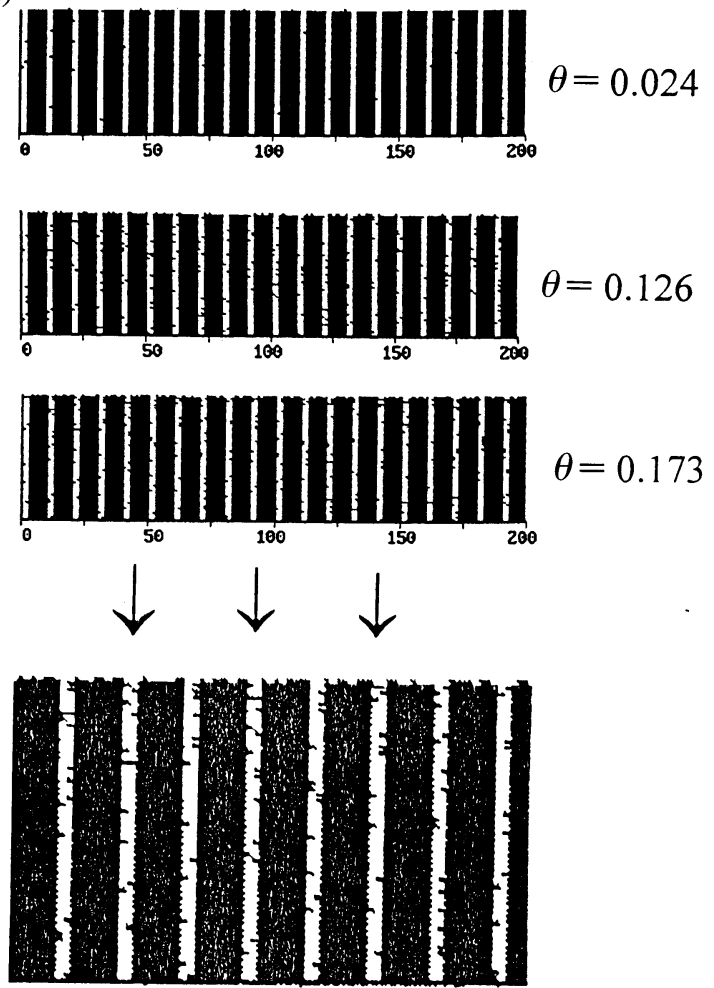

(b)

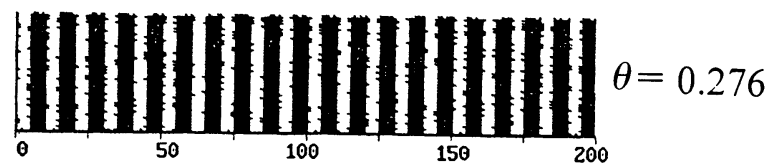

$R=S / S_{\mathrm{o}}$

$S$ and $S_{\mathrm{o}}$ being the number of adsorption sites of length $a$ at a given cs substrate and at the smooth substrate, respectively. The phenomenological relationship between $f(R)$ and $R$ is

$f=K R^{-n}$

where $K \cong 1$ and $n$ depends on $P_{\mathrm{s}}, d$ and $L$. For $P_{\mathrm{s}}=0.98$ and $d=1.6 \times 10^{-7} \mathrm{~cm}$, i.e. when $R$ depends only on pore depth $L$, it results in $n=0.44$ (Fig. 4).

Eq. (4) predicts two limiting cases, for $S \Rightarrow S_{\text {o }}$, $f(R) \Rightarrow 1$, and for $R \Rightarrow \infty, f(R) \Rightarrow 0$. Accordingly, $f(R)=1$ means a full accessibility of the substrate surface for particle adsorption, whereas the reverse situation is given by $f(R) \Rightarrow 0$.

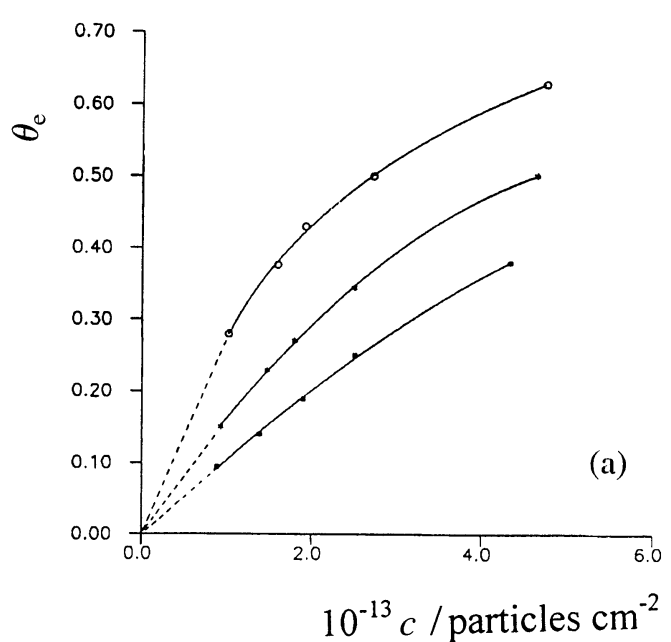

$\mathrm{cm}, d^{\prime}=4.8 \times 10^{-7} \mathrm{~cm}, L=1.5 \times 10^{-6} \mathrm{~cm}, c=2 \times 10^{14}$ particles $\mathrm{cm}^{-2}$, and $P_{\mathrm{s}}=0.98$. For each snapshot the value of $\theta$ is indicated. A zoomed snapshot for $\theta=0.173$ indicating (arrows) some pores where adsorbate pore blocking is produced by either blocking of the pore entrance or a bottleneck effect is shown. (b) Monte Carlo snapshots resulting for $\theta=0.276$ for $d=3.2 \times 10^{-7} \mathrm{~cm}, d^{\prime}=3.2 \times$ $10^{-7} \mathrm{~cm}, L=1.2 \times 10^{-6} \mathrm{~cm}, c=1.1 \times 10^{14}$ particles $\mathrm{cm}^{-2}$, and $P_{\mathrm{s}}=0.98$.

these plots $(\beta)$ increases as $d$ is increased from $1.6 \times$ $10^{-7}$ to $3.2 \times 10^{-7} \mathrm{~cm}$, and decreases with $L$ as it is changed from $8 \times 10^{-8}$ to $4 \times 10^{-6} \mathrm{~cm}$. Seemingly, these results would indicate a dependence of $\Delta G_{\text {ads }}^{\circ}$ on $L$ and $d$, a situation which exceeds the framework of the Langmuir equation. This situation has been taken into account by introducing an empirical surface roughness-dependent correction factor, $f(R)$, defined by the ratio:

$f(R)=\beta / \beta_{\mathrm{s}}$

where $\beta$ and $\beta_{\mathrm{s}}$ are the adsorption coefficients for a given substrate and the smooth substrate, respectively.

The dependence of $f(R)$ on the geometric parameters of the cs substrate derived from Monte Carlo data is expressed in terms of the roughness factor defined as

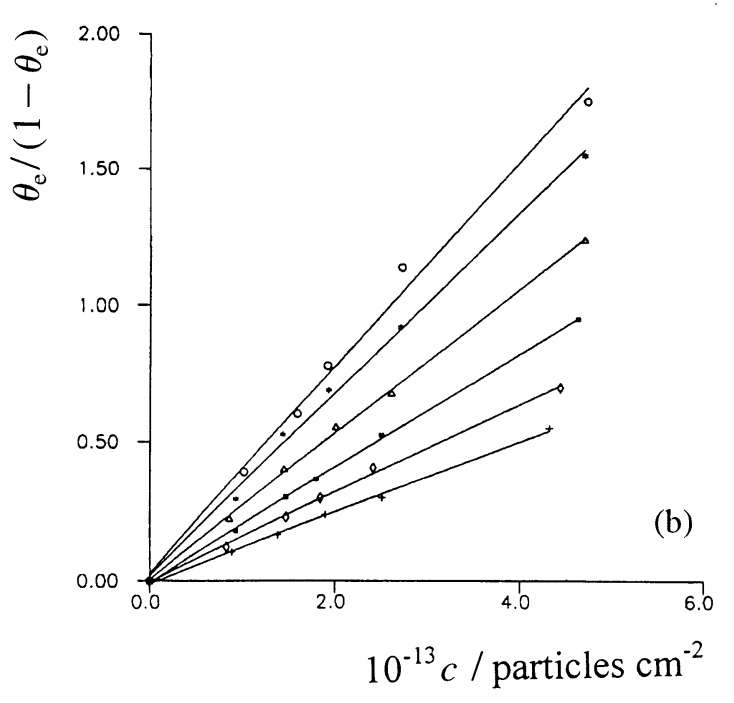

Fig. 3. (a) Plots of $\theta_{\mathrm{e}}$ versus $c$ for $d=1.6 \times 10^{-7} \mathrm{~cm}, d^{\prime}=4.8 \times 10^{-7}$ $\mathrm{cm}, P_{\mathrm{s}}=0.98$ and different values of $L$. (O) $L=0 ;\left({ }^{*}\right) L=2.4 \times$ $10^{-6} \mathrm{~cm}$; ( $\left.\mathbf{\square}\right) L=4 \times 10^{-6} \mathrm{~cm}$. (b) Plots of $\theta_{\mathrm{e}} /\left(1-\theta_{\mathrm{e}}\right)$ versus $c$ for $d=3.2 \times 10^{-7} \mathrm{~cm}, d^{\prime}=3.2 \times 10^{-7} \mathrm{~cm}, P_{\mathrm{s}}=0.98$ and different values of $L$. (○) $L=0$; (*) $L=8 \times 10^{-7} \mathrm{~cm} ;(\triangle) L=1.6 \times 10^{-6} \mathrm{~cm}$; (ם) $L=2.4 \times 10^{-6} \mathrm{~cm} ;(\diamond) L=3.2 \times 10^{-6} \mathrm{~cm} ;(+) L=4 \times 10^{-6}$ $\mathrm{cm}$. 


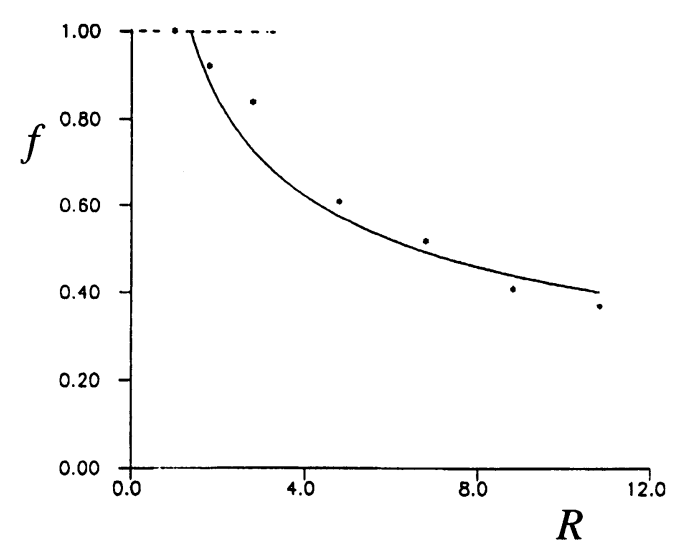

Fig. 4. Plots of $f$ versus $R(L)$ for $P_{\mathrm{s}}=0.98$ and $d=1.6 \times 10^{-7} \mathrm{~cm}$.

\section{The experimental system}

Py adsorption data from $x \mathrm{M} \mathrm{Py}+0.1 \mathrm{M} \mathrm{HClO}_{4}$ aqueous solutions $\left(10^{-3} \leq x \leq 10^{-2}\right)$, at $298 \mathrm{~K}$ on smooth and columnar structured $\mathrm{Au}$ with different $R$ values and applied potentials $(E, \quad$ vs. $\mathrm{Hg}\left|\mathrm{Hg}_{2} \mathrm{SO}_{4}\right| \mathrm{K}_{2} \mathrm{SO}_{4 \text { (sat) }}$ ) greater than the zero charge potential $\left(E_{\mathrm{pzc}}\right)$ [11], were compared to the predictions of the simple model described above. The cs-Au electrodes were prepared by fast electroreduction of a thin hydrous gold oxide layer accumulated by anodization of polycrystalline $\mathrm{Au}$ in $0.5 \mathrm{M} \mathrm{H}_{2} \mathrm{SO}_{4}$ [12]. The cs-Au electrodes have been studied carefully by electrochemi- cal methods $[13,14]$ combined with ellipsometry [15], and STM $[12,16]$. They consisted of a polycrystalline array of nm-sized smooth columns separated by deep pores. The value of $R$ was determined from the $\mathrm{O}$-atom electrosorption voltammograms using a smooth $(R=1)$ $\mathrm{Au}$ surface as reference [17]. As discussed elsewhere [17], the oxygen atom was a yardstick sufficiently small to measure the entire surface area of the cs-Au electrode, thus providing an accurate value of $R$. As observed by in situ STM [18] these electrodes exhibit a roughness relaxation leading to a decrease in the surface area to attain a low surface Gibbs energy. However, cs-Au electrodes, aged for $24 \mathrm{~h}$ in $0.5 \mathrm{M} \mathrm{H}_{2} \mathrm{SO}_{4}$ at $298 \mathrm{~K}$, reached a situation in which change neither in their $R$ nor in their voltammetric features was observed in the time scale used in electroadsorption measurements $[16,18,19]$. For stabilized electrodes, $R$ increases almost linearly with the thickness $(h)$ of the electroreduced gold overlayer covering the range $1 \leq R \leq 50$ (Fig. 5a), the pore depth being proportional to the film thickness. A typical surface of a stabilised cs-Au electrode is shown in Fig. $5 \mathrm{~b}$ for $R=5$. The average diameter was $\left\langle d^{\prime}\right\rangle=1.8 \times 10^{-6} \mathrm{~cm}$ for columns, while pores as small as $d=2 \times 10^{-7} \mathrm{~cm}$ were observed (Fig. $5 \mathrm{c})$. The size of real features could be even smaller when tip-size effects are considered. The column-pore model [12] for cs-Au electrodes accounts for the linear $R$ versus $h$ plot (Fig. 5a) for values of $R$ in the range
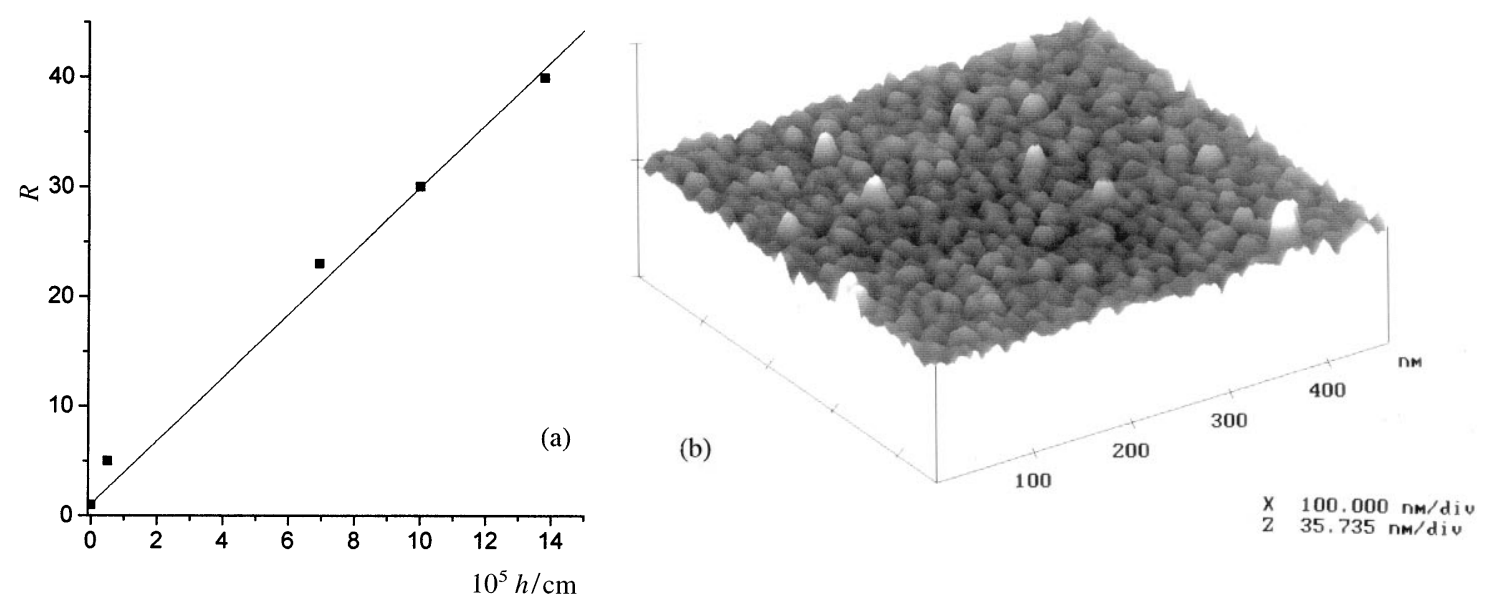

Section Analysis

(c)
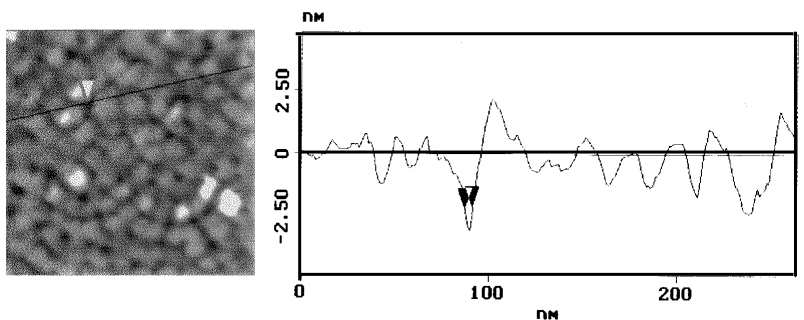

Fig. 5. (a) $R$ versus $h$ plot for stabilized cs-Au electrodes. (b) Three dimensional STM image of a stabilized cs-Au surface, $R=5$. (c) STM image (top view) and cross section showing nanometer size column tips and pores. Arrows indicate a nm-sized pore. 


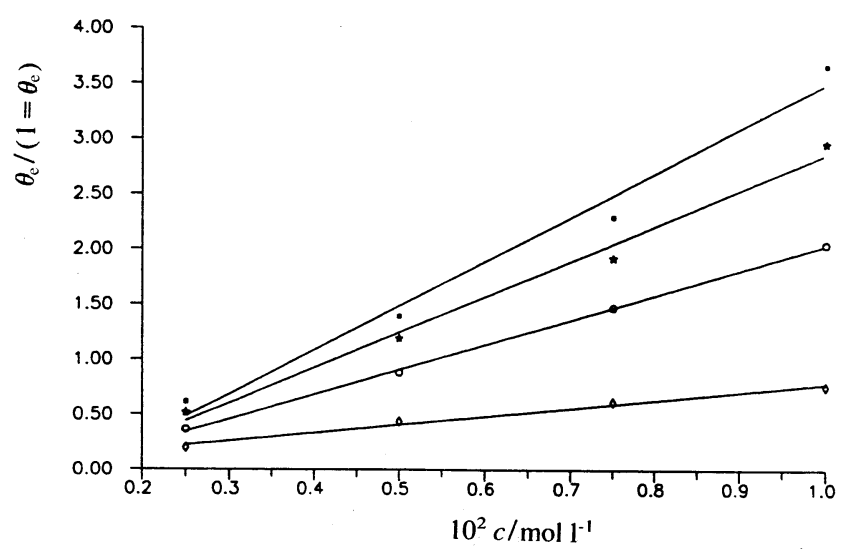

Fig. 6. Plots of $\theta_{\mathrm{e}} /\left(1-\theta_{\mathrm{e}}\right)$ versus $c$ for pyridine adsorption on polycrystalline smooth $(R=1)$ Au electrodes from aqueous $c \mathrm{M}$

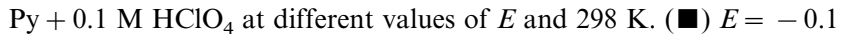
$\mathrm{V}$; (汭 $E=-0.2 \mathrm{~V}$; (○) $E=-0.3 \mathrm{~V}$; ( $) E=-0.4 \mathrm{~V}$.

$1 \leq R \leq 50$. This relationship cannot be explained by a simple roughened and monoatomic stepped surface model. The geometric features of cs-Au electrodes allowed us to compare experimental adsorption data to model predictions. These stabilized electrodes in nonadsorbable electrolytes exhibited a $E_{\mathrm{pzc}}$ value that agreed with that reported for polycrystalline $\mathrm{Au}$ [14].

The surface coverage $\theta$ by Py molecules was estimated from chronocoulometric measurements using stabilized electrodes [11]. The Py adsorption charge density was calculated by dividing the capacitive charge by the real electrode area, the latter estimated from voltammetry by using the $\mathrm{O}$-atom electroadsorption charge. These adsorption data, taken in the range of potential where Py is adsorbed vertically through the $\mathrm{N}$-atom to the Au surface [20], have been interpreted in terms of a Frumkin isotherm. From adsorption data analysis for a smooth $\mathrm{Au}$ electrode $(R=1)$ it was concluded that the value of $\Delta G_{\text {ads }}^{\circ}$ is in the range 20-22 $\mathrm{kJ} \mathrm{mol}^{-1}$, irrespective of $E$. This figure was close to that already reported for Py adsorption on $\mathrm{Au}$ in neutral electrolytes [20,21]. Furthermore, the interaction parameter $\boldsymbol{a}$ from the Frumkin isotherm was small and positive $(\boldsymbol{a} \cong 0.5)$ indicating weak Py-Py attractive interactions [22].

On the other hand, for cs-electrodes the value of $\Delta G_{\text {ads }}^{\circ}$ was close to that found for $R=1$. The value of $\boldsymbol{a}$ changed from -0.5 to -2 as $R$ was increased from 10 to 45. These figures would suggest $\mathrm{Py}-\mathrm{Py}$ repulsive interactions. However, it has been proposed [11] that this difference in the value of $\boldsymbol{a}$ between smooth and rough Au was due mainly to excluded volume effects at pores of rough $\mathrm{Au}$ rather than to a true $\mathrm{Py}-\mathrm{Py}$ repulsive interaction.
The preceding conclusion allowed us to accept a weak Py-Py adsorbate interaction on polycrystalline smooth $\mathrm{Au}$, as has been reported in the literature [22-24]. Accordingly, the corresponding adsorption data could also be represented by a Langmuir isotherm [25]. For this reason, the adsorption of Py on nm-sized cs-Au substrates [12] was chosen as an adequate system for testing the model.

\section{Results and interpretation}

Adsorption data from Ref. [11] were plotted according to a Langmuir isotherm (Fig. 6). Linear $\theta_{\mathrm{e}} /\left(1-\theta_{\mathrm{e}}\right)$ versus $c$ plots for different values of $E$ (vs. $\left.\mathrm{Hg} \mid \mathrm{Hg}_{2} \mathrm{SO}_{4}\right)$ and $R$ were obtained, at least within the error of experimental data. The slope of these lines, $\beta \propto \exp \left(\Delta G_{\text {ads }}^{\circ} / k T\right)$, increases as $E$ is shifted positively. Therefore Eq. (1) is valid to describe the adsorption of Py molecules on $\mathrm{Au}$, irrespective of $R$ (Fig. 7a,b). However, at constant $E$, the value of $\beta$ decreases sharply as $R$ is changed from 1 to 43 . Values of $\beta$ and $\Delta G_{\text {ads }}^{\circ}$ for different values of $R$ and $E$ are shown in Table 1. Values of $\Delta G_{\text {ads }}^{\circ}$ are referred to the unit mole fraction in the bulk of the solution and 0.5 coverage at the surface as the standard state.

The experimental adsorption isotherms on cs-Au substrates behave as shown in Fig. $3 \mathrm{~b}$ because, as already mentioned, pore diameters of the order of $d=(1-2) \times 10^{-7} \mathrm{~cm}$ from STM imaging (Fig. 5) $[11,24]$ and pore lengths $L \gg 10^{-6} \mathrm{~cm}[16]$ have been observed. The pore size distribution function (Fig. 8) is of the form $N \propto d^{-\tau}$ with $\tau=0.65$ [19]. This pore diameter distribution implies that small pores with $d=$ $1-2 \mathrm{~nm}$ dominate in the cs-Au topography being responsible for the distortion of the adsorption isotherm (excluded volume effect). Therefore, the values of $d^{\prime}, d$ and $L$ resulting from cs-Au electrodes (Fig. 5b,c) are of the order of those values used in the Monte Carlo simulation. Furthermore, the non-negligible Py-substrate interaction is consistent with values of $P_{\mathrm{s}}$ used in the simulations. Correspondingly, the decrease in $\beta$ with $R$, in principle, can be assigned to excluded area effects $[11,24]$ that can be accounted for considering the factor $f(R)$, so that in all cases $\beta=f \beta_{\mathrm{s}}$.

Experimental data also show that $f=K R^{-n}$ plots (Fig. 9) result with $K=1 \pm 0.1$ and values of $n$ in the range $0.54-0.79$ depending on $E$ (Table 2). As described in Section 3, the power law behaviour was predicted by the model and supports the assumption that pure geometric effects become responsible for the dependence of $\beta$ on $R$. Values of $f$ in the range $0.05-$ 0.35 and values of $n$ suggest that deep narrow pores $[11,26]$ play a key role in the adsorption behaviour of Py on cs-Au. 

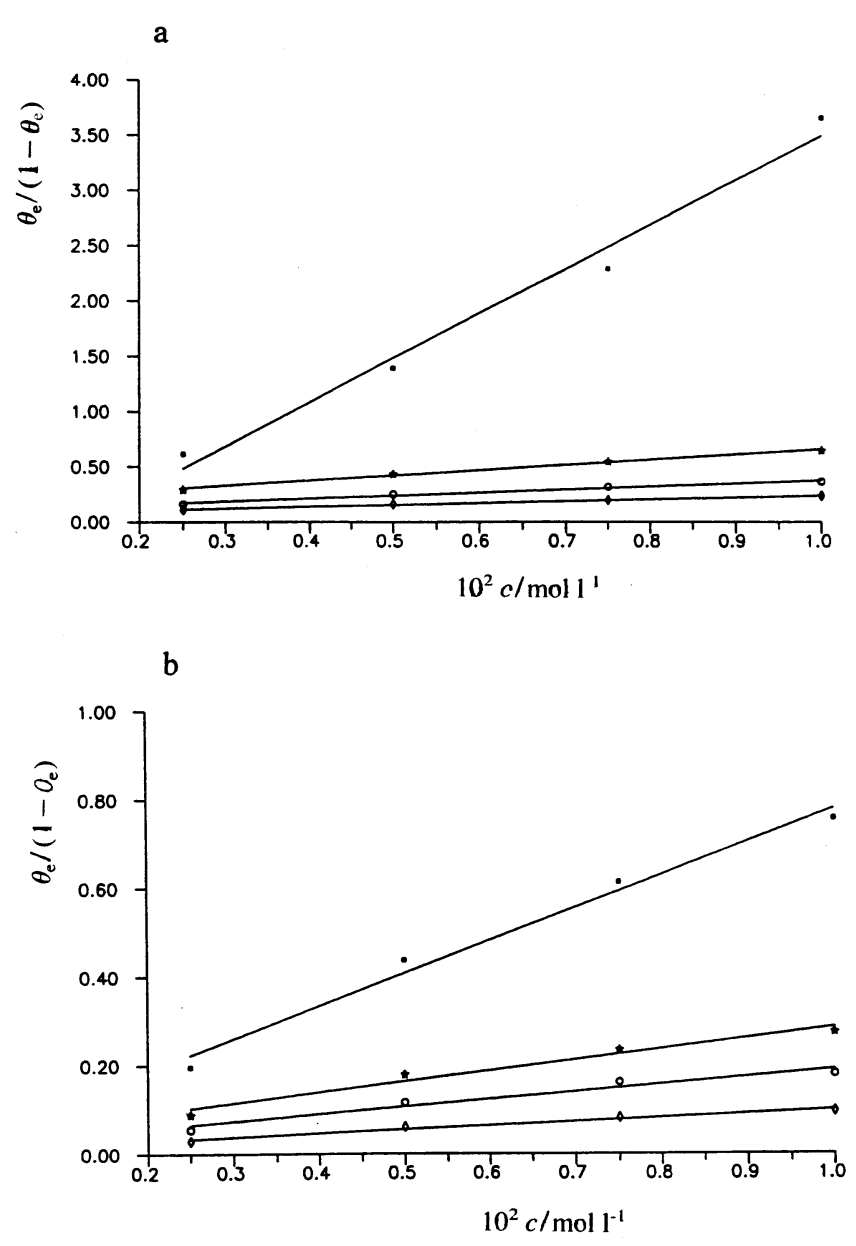

Fig. 7. Plots of $\theta_{\mathrm{e}} /\left(1-\theta_{\mathrm{e}}\right)$ versus $c$ for pyridine adsorption on cs-Au electrodes with different values of $R$ from aqueous $c \mathrm{M} \mathrm{Py}+0.1 \mathrm{M}$ $\mathrm{HClO}_{4}$ and $298 \mathrm{~K}$. (a) $E=0 \mathrm{~V}$ and (घ) $R=1 ;(*) R=10 ;(\bigcirc)$ $R=22 ;(\diamond) R=43$. (b) $E=-0.4 \mathrm{~V}$ and (口) $R=1 ;(\star) R=10 ;(\bigcirc)$ $R=22 ;(\diamond) R=43$.

The tendency of $n$ with $E$ can be explained considering the slight increase in the surface concentration $(\Gamma)$ of Py on polycrystalline Au with $E$ in the range $E>$ $E_{\mathrm{pzc}}$ [23]. Then, the increase in $\Gamma$ results in an increase in the number of blocked pores, and accordingly, $n \rightarrow 1$ (Table 2) and $f \rightarrow 0$ (Fig. 10). A scheme of Py adsorbate configuration for $E>E_{\mathrm{pzc}}$ at smooth domains [25-27]

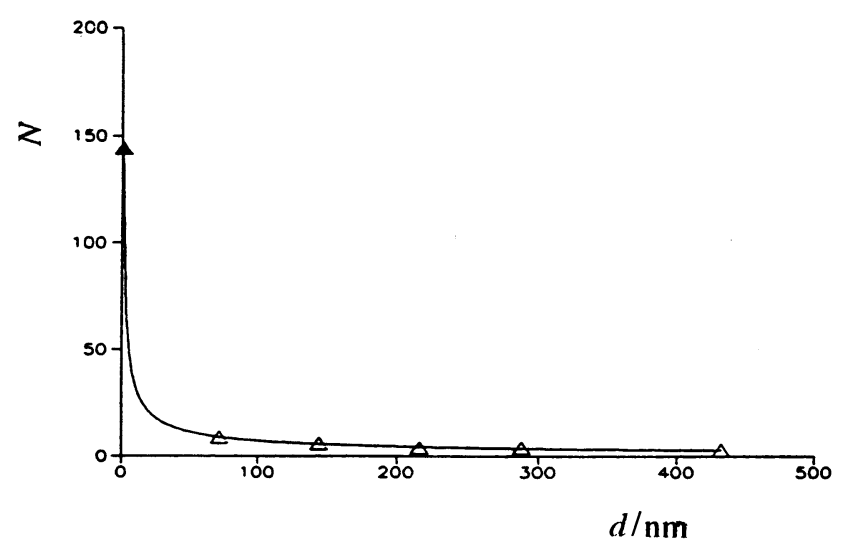

Fig. 8. Plot of the pore size distribution function. $(\triangle)$ Experimental data from $1200 \times 1200 \mathrm{~nm}^{2}$ STM images. The solid line represents the best fit of experimental data with $N=142.6 d^{-0.65}$; $(\boldsymbol{\Delta})$ denotes the number of pores calculated from this equation for $d=1 \times 10^{-7} \mathrm{~cm}$, which is the smallest pore size derived from STM imaging data $[9,13]$ (lower cut off).

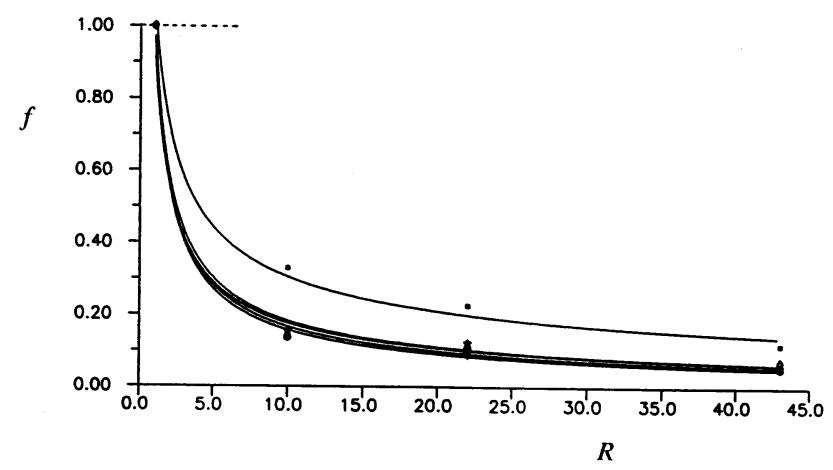

Fig. 9. Plot of $f$ versus $R$ for pyridine adsorption on cs-Au electrodes from aqueous $c \mathrm{M} \mathrm{Py}+0.1 \mathrm{M} \mathrm{HClO}_{4}$ and $298 \mathrm{~K}$ for different values of $E$. (囬) $E=-0.4 \mathrm{~V}$; (它) $E=-0.3 \mathrm{~V} ;(\bigcirc) E=-0.2 \mathrm{~V}$; $(\diamond)$ $E=-0.1 \mathrm{~V} ;\left(^{*}\right) E=0.0 \mathrm{~V}$.

and nm-sized pores is shown in Fig. 11a. From this scheme it can be envisaged that for $E<E_{\mathrm{pzc}}$ the change in the adsorbate configuration from vertical to parallel would result in a lower pore blocking effect (Fig. 11b).

In conclusion, the agreement between experimental data and the predictions of the simple model indicates

Table 1

Values of $\beta$ and apparent $\Delta G_{\mathrm{ad}}^{\circ}$ derived from Py adsorption on cs-Au electrodes at $298 \mathrm{~K}$ for different values of $R$ and $E$.

\begin{tabular}{|c|c|c|c|c|c|c|c|c|c|c|}
\hline \multirow[t]{2}{*}{$\mathrm{R}$} & \multicolumn{10}{|l|}{$E / \mathrm{V}$} \\
\hline & \multicolumn{2}{|l|}{-0.4} & \multicolumn{2}{|l|}{-0.3} & \multicolumn{2}{|l|}{-0.2} & \multicolumn{2}{|l|}{-0.1} & \multicolumn{2}{|l|}{0} \\
\hline 1 & 4108 & -20.6 & 12480 & -23.4 & 17830 & -24.2 & 21120 & -24.7 & 21095 & -24.7 \\
\hline 10 & 1352 & -17.9 & 1954 & -18.8 & 2438 & -19.3 & 2956 & -19.8 & 2977 & -19.8 \\
\hline
\end{tabular}


the importance of excluded area effects at nm-sized pores for the Py adsorption on cs-Au surfaces. The model accounts for results obtained over a relatively wide range of applied potentials, thus providing the justification and usefulness of the Monte Carlo simula-

Table 2

Values of $K$ and $n$ derived from Py adsorption on cs-Au electrodes at $298 \mathrm{~K}$

\begin{tabular}{lll}
\hline$E / \mathrm{V}$ & $K$ & $n$ \\
\hline-0.4 & 1.05 & 0.54 \\
-0.3 & 0.97 & 0.73 \\
0.2 & 0.96 & 0.76 \\
-0.1 & 0.91 & 0.71 \\
0.0 & 0.96 & 0.79 \\
\hline
\end{tabular}

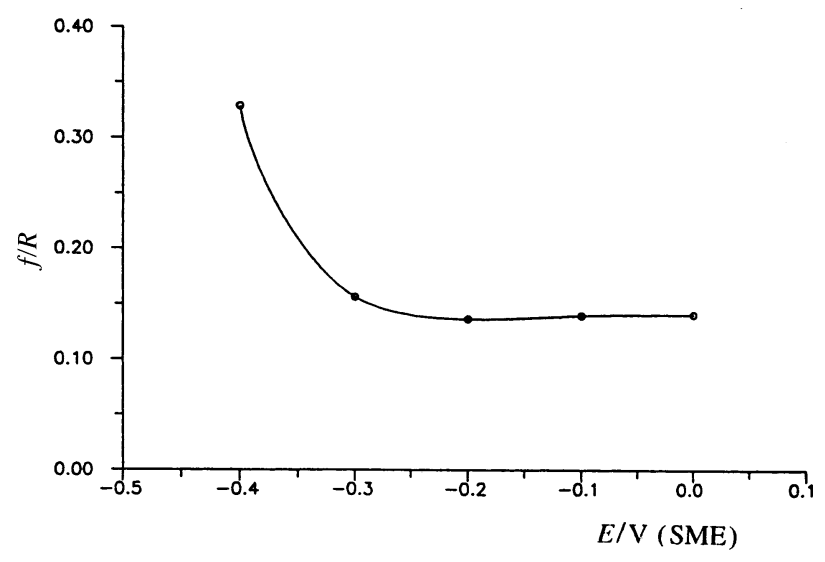

Fig. 10. Plot of $f(R)$ versus $E$ for a cs-Au electrode with $R=10$.

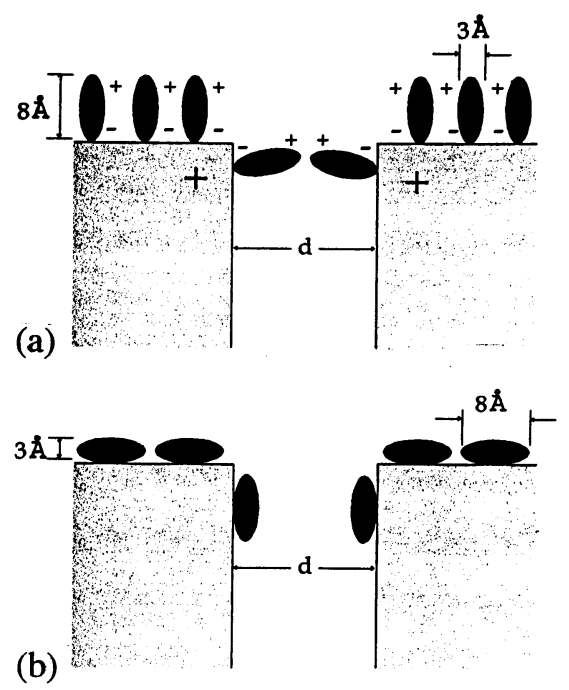

Fig. 11. Schemes of adsorbed Py molecules at pore entrances. (a) Vertical adsorbate configuration $\left(E>E_{\mathrm{pzc}}\right)$; (b) Parallel adsorbate configuration $\left(E<E_{\mathrm{pzc}}\right)$. tion for the analysis of molecular adsorption at irregular solid surfaces.

\section{Acknowledgements}

This work was partially supported by the Consejo Nacional de Investigaciones Científicas y Técnicas (CONICET) (PIP 4376 and PIP 0897/98) and Agencia Nacional de Promoción Científica y Tecnológica (PICT 97-1993) from Argentina and Proyecto $N^{\circ}$ PB97-0035, DGICYT from Spain.

\section{References}

[1] M. Jaroniec, R. Madey, Physical Adsorption on Heterogeneous Solids, Elsevier, Amsterdam, 1988.

[2] D. Avnir (Ed.), The Fractal Approach to Heterogeneous Chemistry, Wiley, New York, 1989.

[3] W. Rudzinski, D.H. Everett, Adsorption of Gases on Heterogeneous Surfaces, Academic Press, London, 1992, Chs. 4-6, pp. 83-199.

[4] A. Seri-Levi, D. Avnir, Langmuir 9 (1993) 3067; 3073.

[5] A Seri-Levi, D. Avnir, Proc. IVth Int. Conf. on Fundamentals of Adsorption, Kyoto, 1992, International Adsorption Society, 1993, p. 365.

[6] P. Pfeifer, Y.J. Wu, M.W. Cole, J. Krim, Phys. Rev. Lett. 62 (1989) 1997.

[7] L.D. Gelb, K.E. Gubbies, Langmuir 14 (1998) 2097.

[8] V.A. Bakaev, W.A. Steele, Langmuir 13 (1997) 1054.

[9] M.M. Gomez, J.M. Vara, J.C. Hernandez, R.C. Salvarezza, A.J. Arvia, Electrochim. Acta 44 (1998) 1255.

[10] V.K. Dobruskin, Langmuir 14 (1998) 3840, 3847.

[11] M.M. Gómez, M.P. García, J. San Fabián, L. Vázquez, R.C. Salvarezza, A.J. Arvia, Langmuir 13 (1997) 1317.

[12] L. Vázquez, A. Bartolomé, A.M. Baró, C. Alonso, R.C. Salvarezza, A.J. Arvia, J. Surf. Sci. 215 (1989) 171.

[13] M.E. Vela, R.C. Salvarezza, A.J. Arvia, Electrochim. Acta 35 (1990) 117.

[14] M.P. Gómez, M.M. Gómez, R.C. Salvarezza, A.J. Arvia, J. Electrochem. Soc. 141 (1994) 2291.

[15] M.E. Vela, J.O. Zerbino, A.J. Arvia, Thin Solid Films 233 (1993) 82.

[16] R.C. Alonso, J.M. Salvarezza, A.J. Vara, L. Arvia, A. Vazquez, A. Bartolomé, A.M. Baró, J. Electrochem. Soc. 137 (1990) 2161.

[17] R.C. Salvarezza, A.J. Arvia, in: B.E. Conway, J.O’M. Bockris, R.E. White (Eds.), Modern Aspects of Electrochemistry, vol. 28, Plenum Press, New York, 1996, Ch. 5, p. 289.

[18] G. Andreasen, M. Nazzarro, J. Ramirez, R.C. Salvarezza, A.J. Arvia, J. Electrochem. Soc. 143 (1995) 466.

[19] J.M. Gómez-Rodríguez, L. Vázquez, A.M. Baró, R.C. Salvarezza, J.M. Vara, A.J. Arvia, J. Phys. Chem. 96 (1992) 347.

[20] J. Lipkowski, L. Stolberg, in: J. Lipkowski, P. Ross (Eds.), Adsorption of Molecules at Metal Electrodes, VCH, New York, 1992, p. 171 and references therein.

[21] L. Stolberg, J Richer, J. Lipkowski, J. Electroanal. Chem. 207 (1986) 213.

[22] L. Stolberg, S. Morin, J. Lipkowski, D.E. Irish, J. Electroanal Chem. 307 (1991) 241. 
[23] L. Stolberg, J. Lipkowski, D.E. Irish, J. Electroanal Chem. 322 (1992) 357.

[24] J. Lipkowski, L. Stolberg, D.F. Yang, B. Pettinger, S. Mirwald, F. Henglein, D.M. Kolb, Electrochim. Acta 39 (1994) 1045.

[25] M.M. Gómez, M.P. García, J. San Fabián, L. Vázquez, R.C.
Salvarezza, A.J. Arvia, Langmuir 12 (1996) 818.

[26] G. Andreasen, M.E. Vela, R.C. Salvarezza, A.J. Arvia, Langmuir 13 (1997) 6814.

[27] G. Andreasen, M.E. Vela, R.C. Salvarezza, A. Arvia, J. Electroanal. Chem. 467 (1999) 230. 